\title{
Effects of Sirtuin 1 on the proliferation and osteoblastic differentiation of periodontal ligament stem cells and stem cells from apical papilla
}

\author{
Q.-B. Zhang ${ }^{1,2 *}$, W. Cao ${ }^{1 *}$, Y.-R. Liu ${ }^{3}$, S.-M. Cui ${ }^{2}$ and Y.-Y. Yan ${ }^{1}$ \\ ${ }^{1}$ Key Laboratory of Stomatology, Guangzhou Medical University, \\ Guangzhou City, Guangdong Province, China \\ 2Department of Temporo-Mandibular Joint Surgery, Hospital of Stomatology, \\ Guangzhou Medical University, Guangzhou, Guangdong Province, China \\ ${ }^{3}$ Department of Pediatric Dentistry, Hospital of Stomatology, \\ Guangzhou Medical University, Guangzhou, Guangdong Province, China \\ *These authors contributed equally to this study. \\ Corresponding author: Q.-B. Zhang \\ E-mail: doctorqingbin@hotmail.com
}

Genet. Mol. Res. 15 (1): gmr.15015234

Received August 7, 2015

Accepted October 19, 2015

Published March 24, 2016

DOI http://dx.doi.org/10.4238/gmr.15015234

ABSTRACT. The function of SIRT1 in the proliferation and osteoblastic differentiation of dental stem cells is unclear. The aim of this study was to assess the roles of SIRT1 in these processes using periodontal ligament stem cells (PDLSCs) and stem cells from apical papilla (SCAPs). A defined concentration of resveratrol, an SIRT1 activator, or nicotinamide, an SIRT1 inhibitor, was administered to PDLSCs, SCAPs, and a mixed group of the two cell lines, and their effects on these processes analyzed. Cell proliferation was tested using microtitration with a tetrazolium dye (MTT). Alkaline phosphatase (ALP) activity, mineralization ability, and the expression of osteoblastic differentiation-associated genes were assessed as well. These studies demonstrated that resveratrol could 
promote cell proliferation of all three groups in a gradually increasing trend over time. In contrast, nicotinamide suppressed the proliferation of the three cell lines. The results also showed that the markers of osteoblastic differentiation: ALP activity, mineralization ability, and the expression levels of the osteoblastic genes $A L P$, osteopontin, osteocalcin, and bone sialoprotein, were enhanced in the groups with resveratrol treatment. In contrast, following addition of nicotinamide, ALP activity, mineralization ability, and the expression levels of the osteoblastic genes were downregulated in the cells. Together, these results suggest that the SIRT1 activator and inhibitor compounds, resveratrol and nicotinamide, function at high efficiency in adjusting cell proliferation, and that SIRT1 is a powerful regulator of osteoblastic differentiation of PDLSCs and SCAPs. In addition, co-culture of the two cell lines could promote their abilities of proliferation and osteogenic differentiation.

Key words: Sirtuin1; Cell culture; Periodontal ligament stem cells; Stem cells from apical papilla; Proliferation; Osteoblastic differentiation

\section{INTRODUCTION}

To date, reports have verified that mesenchymal stem cells exist in odontogenic tissues, such as periodontal ligament, endodontium, apical papilla, and odontotheca (Seo et al., 2004; Hayami et al., 2007). These special cell populations have been called dental stem cells. Many authors have reported that dental stem cells could be isolated, cultivated, and proliferated under certain conditions (Beertsen et al., 1997; Sonoyama et al., 2007). However, little is known about which cell type acts as the origin of dental stem cells and how they differentiate into functional cells. Certain dental stem cells [periodontal ligament stem cells (PDLSCs) and stem cells from apical papilla (SCAPs)] have been verified to have variable differentiation potentials and thus can be used in oral tissue engineering (Sumita et al., 2006; Moshaverinia et al., 2014). The osteoblastic differentiation abilities of PDLSCs and SCAPs have been confirmed and their functions in promoting osteogenesis have also been verified in multiple studies (Cate, 1996; Yang et al., 2009). The purpose of periodontal therapy is to regenerate and restore various periodontal components affected by disease to their original form, function, and consistency (Murakami et al., 1999). To achieve periodontal regeneration, new therapeutic approaches currently available include guided tissue regeneration, application of signaling molecules (Thesleff and Mikkola, 2002), and stem cell transplantation. However, the effectiveness of these methods is not as optimistic as might be imagined.

Silent information regulator1 (Sirtuin1, SIRT1: a member of the Sirtuin family) is a homolog of the SIRT2 protein. SIRT1 and SIRT2 belong to Sirtuin family, both of them can remove acetyl; SIRT2 was studied in saccharomycetes and nematode, not in mammal, but SIRT1 was discovered in mammal (Blander et al., 2009). SIRT1 is a nicotinamide adenosine dinucleotide-dependent class III histone deacetylase (Hisahara et al., 2008), and plays a central role in regulating gene transcription, energy metabolism, and cell senescence in vivo, and especially in the regulation of glucose metabolism, lipid metabolism, and insulin secretion (Picard et al., 2004). As a histone deacetylase, SIRT1 regulates various physiological processes by activating p53, Foxo, and other functional proteins in vivo. Further studies have shown that SIRT1 regulates cellular differentiation 
and control metabolic pathways in a wide variety of tissues (Bai et al., 2008; Pillarisetti, 2008). Unfortunately, the consequences of SIRT1 over- or under-expression remain little known. Recently, the SIRT1 activator, resveratrol, was shown to inhibit the differentiation of pig preadipocytes, whereas nicotinamide, an SIRT1 inhibitor, markedly stimulated the proliferation and differentiation of these cells (Fulco et al., 2003; Bäckesjö et al., 2007).

However, apart from the report of the effects of SIRT1 on the osteoblastic differentiation of human PDLSCs from Lee et al. (2011), the function of SIRT1 in dental stem cells has been rarely reported. Therefore, the aims of this study were to investigate the role of SIRT1 in the proliferation and osteogenic differentiation of human PDLSCs and SCAPs, to clarify its mechanism in osteogenic differentiation, and to provide the experimental basis for clinical utilization of the dental stem cell lines studied.

\section{MATERIAL AND METHODS}

\section{Material}

\section{Acquisition of fresh teeth}

Third molars selected for extraction for orthodontic purpose in adolescents aged 12 to 17 years were utilized in this study. This study was approved by the Hospital of Stomatology, Guangzhou Medical University. Informed consent was obtained from all patients in the study group before performing the research. The freshly extracted teeth were soaked in $1 \mathrm{X}$ phosphate-buffered saline $(5 \mathrm{~mL})$ prior to use.

\section{Tissue}

Periodontal ligament and tissue from apical papilla were obtained from the selected teeth within $4 \mathrm{~h}$ after extraction. Periodontal ligament was gently separated from the surface. A third on the middle of root and apical papilla tissue was gently separated from apical part of the root. Then, both of the tissues are digested separated in a solution of $3 \mathrm{mg} / \mathrm{mL}$ collagenase type I (Worthington Biochem, Freehold, NJ, USA) and $4 \mathrm{mg} / \mathrm{mL}$ dispase (Roche, Mannheim, Germany) for $1 \mathrm{~h}$ at $37^{\circ} \mathrm{C}$. Tissues after digestion were seeded on 10-cm culture dishes (Costar, Cambridge, MA, USA) with alpha modification of Eagle's medium (GIBCO BRL, Grand Island, NY, USA) supplemented with $15 \%$ fetal calf serum and then incubated at $37^{\circ} \mathrm{C}$ in $5 \%$ carbon dioxide (Seo et al., 2004). Sub-culture was started when cells reached $80 \%$ confluence, at approximately 14 days following initial plating.

\section{Confirmation of stem cell identities}

Stem cells were obtained from periodontal ligament cells and their identity as PDLSCs was confirmed by immunohistochemical detection of the mesenchymal stem cell markers antiCD146, anti-CD24 and anti-STRO-1 (Mouse antibodies) (Seo et al., 2004). Mouse antibodies stem cells were also obtained from cell culture of human lower third molars, and their identity as SCAPs was confirmed by immunohistochemical detection of the mesenchymal stem cell markers STRO-1 and CD24 (Seo et al., 2004). 


\section{Methods}

\section{Cell culture and drug application}

Osteogenic induction medium was supplemented with $\beta$-glycerophosphate $(10 \mathrm{mM})$, ascorbic acid $(100 \mu \mathrm{g} / \mathrm{mL})$ and dexamethasone $(10 \mathrm{nM})$. We chose P3 cells after sub-cultural as working cells. Cells were seeded into 18 holes of 24 -well plates at $1 \times 10^{5}$ cells/hole, and 6 holes were randomly selected as the control group. The defined concentration of resveratrol and nicotinamide (Lee et al., 2011) utilized was $5 \mathrm{mM}$. Resveratrol and nicotinamide were added to the three groups: PDLSCs, SCAPs and mixed cells, and cells were cultured for 14 days.

\section{Cell proliferation assay}

Cell proliferation was observed by microtitration with tetrazolium dye (3-(4,5-dimethylthiazol2-yl)-2,5-diphenyltetrazolium bromide) (MTT) assay. Briefly, PDLSCs or SCAPs were seeded on 96-well culture plates for $24 \mathrm{~h}$. The cells were then incubated with $5 \mathrm{mM}$ resveratrol or nicotinamide for various times. An MTT solution (Sigma, St. Louis, MO, USA) was then added to the cells, and the cells were incubated for $4 \mathrm{~h}$ at $37^{\circ} \mathrm{C}$. DMSO was then added, and the plates were read on an automated microplate spectrophotometer (Bio-Rad, Berkeley, CA, USA) at $490 \mathrm{~nm}$. The inhibition rate was calculated as follows: ( 1 - OD value of treated cells / OD value of control cells) $\times 100 \%$.

\section{Alkaline phosphatase (ALP) activity assay}

ALP is considered to be an early marker of osteoblastic differentiation. The cells of the six groups (three groups treated with each of the two compounds) were prepared and ALP activity was detected with an ALP assay kit (Jiancheng Co., Nanjing, China) according to the manufacturer instructions. Briefly, cellular protein was extracted using protein lysis buffer containing $50 \mathrm{mM}$ Tris- $\mathrm{HCl}, 150 \mathrm{mM} \mathrm{NaCl}$, and 1\% NP-40. Cells were incubated following application of the phenol standard liquid, buffer, and the substrate solution for $15 \mathrm{~min}$ at $37^{\circ} \mathrm{C}$. After addition of the colorsubstrate solution, reactions were monitored at $520 \mathrm{~nm}$ wavelength with an enzyme micro-plate reader (Jiancheng Co., Nanjing, China). Repeat experiments were performed at least 3 times (e.g., ALP assay).

\section{Alizarin red staining}

After incubation following drug treatment in osteogenic induction medium for 14 days, cell mineralization was determined using Alizarin red staining. Cells were washed twice in distilled water and fixed by incubation in ice-cold $70 \%(\mathrm{v} / \mathrm{v})$ ethanol for $1 \mathrm{~h}$. Cells were then rinsed twice in deionized water and stained with Alizarin red (Sigma-Aldrich) (previously dissolved in deionized water, $\mathrm{pH} 4.2$, at a concentration of $2 \%$ ) for $10 \mathrm{~min}$ at room temperature. Following staining, excess dye was removed in running water. To determine the optical density of the Alizarin red staining, each well was destained for 30 min using $500 \mathrm{~mL} \mathrm{10 \%} \mathrm{cetylpyridinium} \mathrm{chloride} \mathrm{monohydrate}$ (Acros Organics, Morris Plains, NJ, USA), and the absorbance measured at $540 \mathrm{~nm}$ on an ELISA plate reader (Bio-Rad Laboratories). The intensity of Alizarin red staining was analyzed by optical density measurement. 


\section{Quantitative real-time polymerase chain reaction ( $q R T-P C R)$}

Following 14 days in culture, total RNA was isolated using TRIzol reagent (Invitrogen, Carlsbad, CA, USA) according to the manufacturer instructions. RNA (5 $\mu \mathrm{g})$ was then reverse transcribed using AccuPower RT Premix (Bioneer, Daejon, Korea). PCR amplification of the resulting cDNA samples was performed using a GeneAmp PCR system 2400 thermal cycler (PerkinEImer, Wellesley, MA, USA). The primers used are shown in Table 1. PCR conditions were as follows: 30 cycles of denaturation at $94^{\circ} \mathrm{C}$ for $30 \mathrm{~s}$ and annealing at $56^{\circ} \mathrm{C}$ for $30 \mathrm{~s}$. The PCR products were resolved on 1 to $2 \%$ agarose gels and stained with ethidium bromide. Each assay was run in triplicate. All samples were normalized to GAPDH. A comparison was done for each sample using a relative cycle threshold $(\mathrm{Ct})$ comparison. An average $\mathrm{Ct}$ was calculated for the triplicate reactions and normalized to GAPDH $\left(\Delta \mathrm{Ct}=\mathrm{Ct}_{\text {Sample }}-\mathrm{Ct}_{\mathrm{GAPDH}}\right)$. The $\mathrm{Ct}$ values were then compared between different treatments and time points $(\Delta \Delta \mathrm{Ct})$ with normalization to the untreated samples. Finally, a fold-change was calculated from the $\Delta \Delta \mathrm{Ct}$ (fold-change $=2^{\Delta \Delta \mathrm{Ct}}$ ).

Table 1. Osteoblastic gene primers for quantitative real-time PCR.

\begin{tabular}{|c|c|}
\hline Name (amplicon size) & Primers \\
\hline \multirow{2}{*}{ ALP (475 bp) } & 5'-ACG TGG CTA AGA ATG TCA TC-3' \\
\hline & 5'-CTG GTA GGC GAT GTC CTT A-3' \\
\hline \multirow{2}{*}{ Osteopontin (347 bp) } & 5'-CCA AGT AAG TCC AAC GAA AG-3' \\
\hline & 5'-GGT GAT GTC CTC GTC TGT A-3' \\
\hline \multirow{2}{*}{ Osteocalcin (310 bp) } & 5'-CAT GAG AGC CCT CAC A-3' \\
\hline & 5'-AGA GCG ACA CCC TAG AG-3' \\
\hline \multirow{2}{*}{ Bone sialoprotein (746 bp) } & 5'-GTG AAT TCA CCT TTC AGC CCC CAA AAC C-3' \\
\hline & 5'-TGG GAT CCC AGC TGT GAA TGG GCT TCT T-3' \\
\hline
\end{tabular}

\section{Statistical analysis}

Data were statistically analyzed by the SPSS 13.0 software (SPSS, Chicago, IL, USA). The data among groups were compared with the non-parametric test. $\mathrm{P}<0.05$ was deemed to indicate statistical significance.

\section{RESULTS}

\section{Morphologic and histologic examination of stem cell characteristics}

During generation of PDLSCs, microscopic examination revealed that cells became clonogenic 7 to 14 days after primary cell culture, in a fusiform shape with small size (Figure 1A). During generation of SCAPs, cells were observed to have adhered to the culture flask shortly after plating, and reached 80 to $90 \%$ confluence after 2 weeks. Cell appearance was generally fusiform, with 1 to 2 fibroblast-like protuberances; however, the occasional polygonal cell was also observed. Clone formation was characteristic of SCAPs (Figure 1B).

To verify the identities of the generated stem cell lines, the third subculture generation of cells from periodontal ligament was tested by immunofluorescence staining for mesenchymal stem cell markers, with the results showing positive staining for STRO-1 (Figure 2A) and CD146 (Figure 2B) expression in clonogenic cells, which confirmed the stemness of these cells and their identity as PDLSCs. Similarly, cells from apical papilla were positive by immunofluorescence for 
the expression of STRO-1 (Figure 3A) and CD24 (Figure 3B), confirming the identity of these cells as SCAPs.
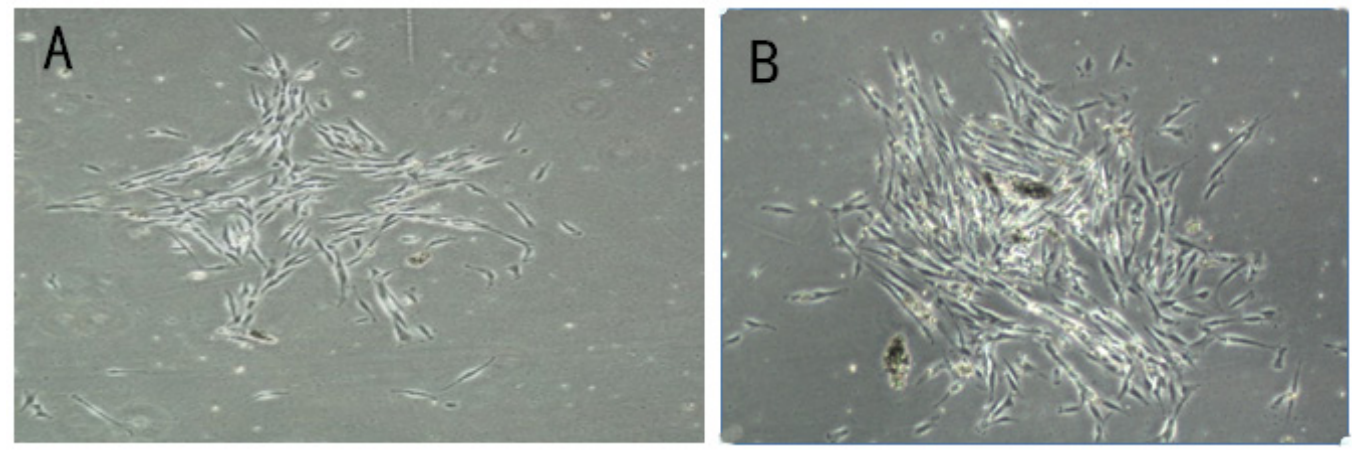

Figure 1. Clone-like appearance of $(\mathbf{A})$ periodontal ligament stem cells (100X); and (B) stem cells from apical papilla (100X).
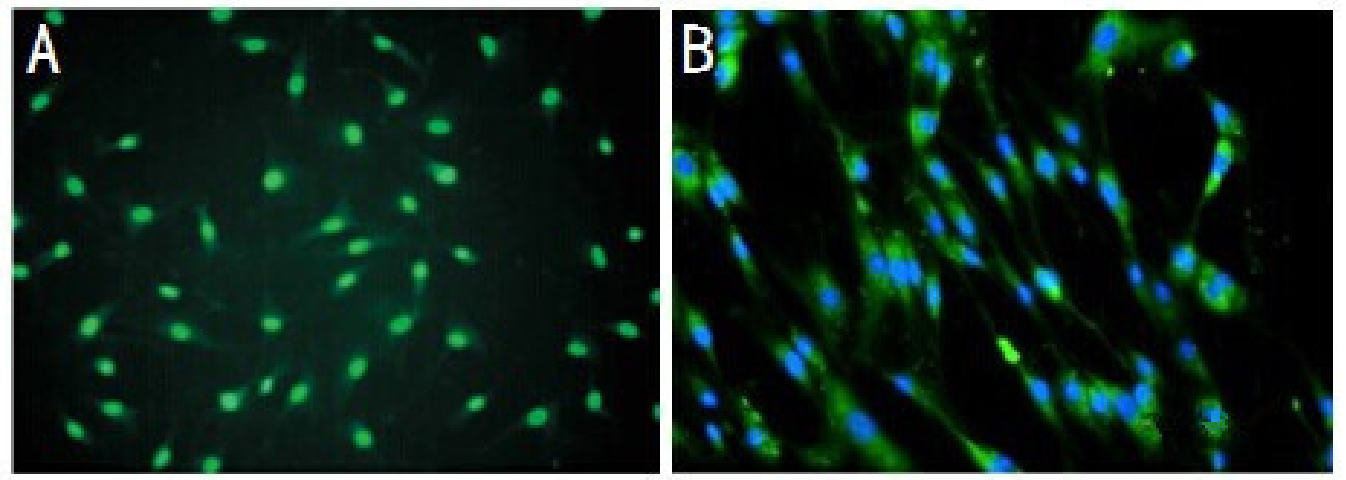

Figure 2. Immunofluorescence analysis of PDLSCs in the third subculture generation (200X). A. STRO-1-positive staining (green). B. CD146-positive staining (green and blue). PDLSCs, periodontal ligament stem cells.
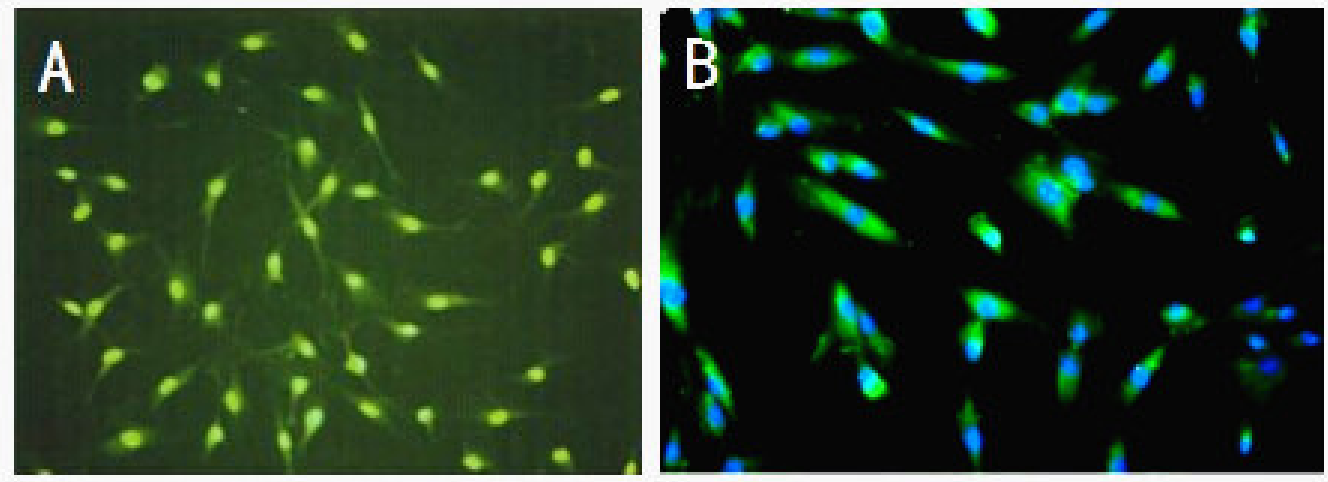

Figure 3. Immunofluorescence analysis of SCAPs in the third subculture generation (200X). A. STRO-1-positive staining (green). B. CD24-positive staining. SCAPs, stem cells from apical papilla (green and blue). 


\section{Cell proliferation as measured by MTT assay}

Following resveratrol treatment, cells in the mixed group showed the highest proliferation property as measured by MTT, followed by the SCAP $(P<0.05)$ and then the PDLSC groups (Figure 4). For nicotinamide-treated cells, those in the control group showed the highest proliferation, followed by the mixed, SCAP, and PDLSC groups, in descending order ( $P<0.05$; Figure 5).

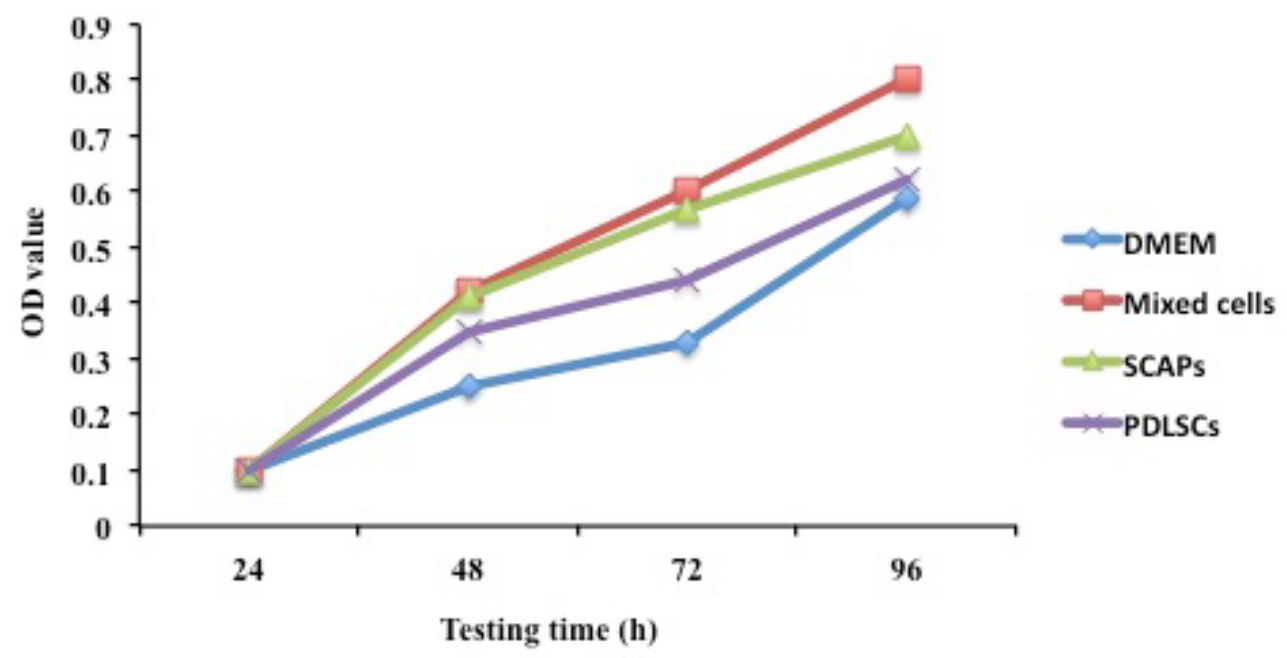

Figure 4. Cell proliferation in the four cell groups with resveratrol treatment. DMEM, Dulbecco's modified Eagle's medium; PDLSCs, periodontal ligament stem cells; SCAPs, stem cells from apical papilla.

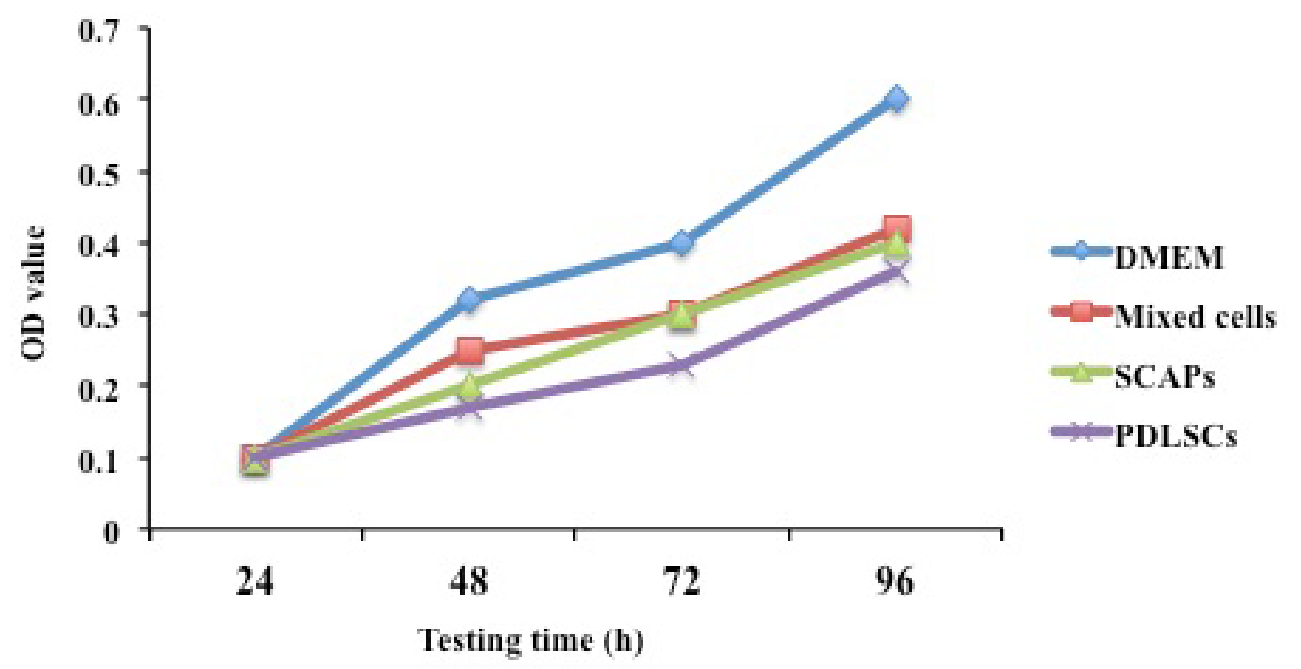

Figure 5. Cell proliferation in the four cell groups with nicotinamide treatment. DMEM, Dulbecco's modified Eagle's medium; PDLSCs, periodontal ligament stem cells; SCAPs, stem cells from apical papilla. 


\section{ALP activity and differentiation among the cell groups}

ALP represents an early marker for dental mesenchymal cell differentiation towards the cemento/osteoblastic phenotype (Seo et al., 2004). In this study, induction of the various cell groups with SIRT1 activator or inhibitor after 4, 8, or 12 days resulted in different expression of ALP activity $(P<0.05)$. ALP activity increased gradually during the first 12 days and reached a maximum in each group (Figure 6). However, in the cell groups treated with SIRT1, ALP expression was stronger than in those treated with SIRT1 inhibitor $(P<0.05$; Figure 7$)$.

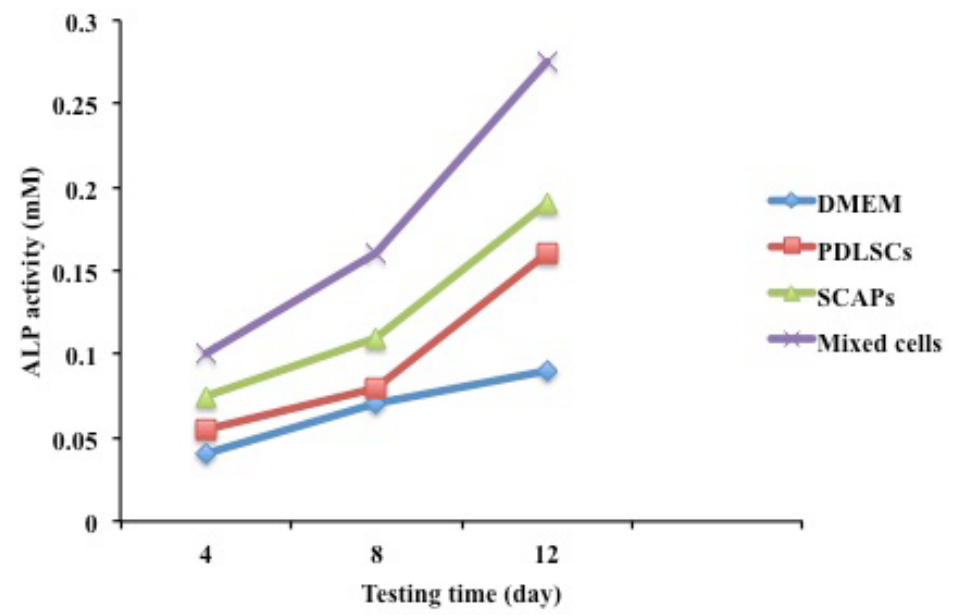

Figure 6. Alkaline phosphatase activities of the cell groups influenced by resveratrol. DMEM, Dulbecco's modified Eagle's medium; PDLSCs, periodontal ligament stem cells; SCAPs, stem cells from apical papilla; ALP, alkaline phosphatase.

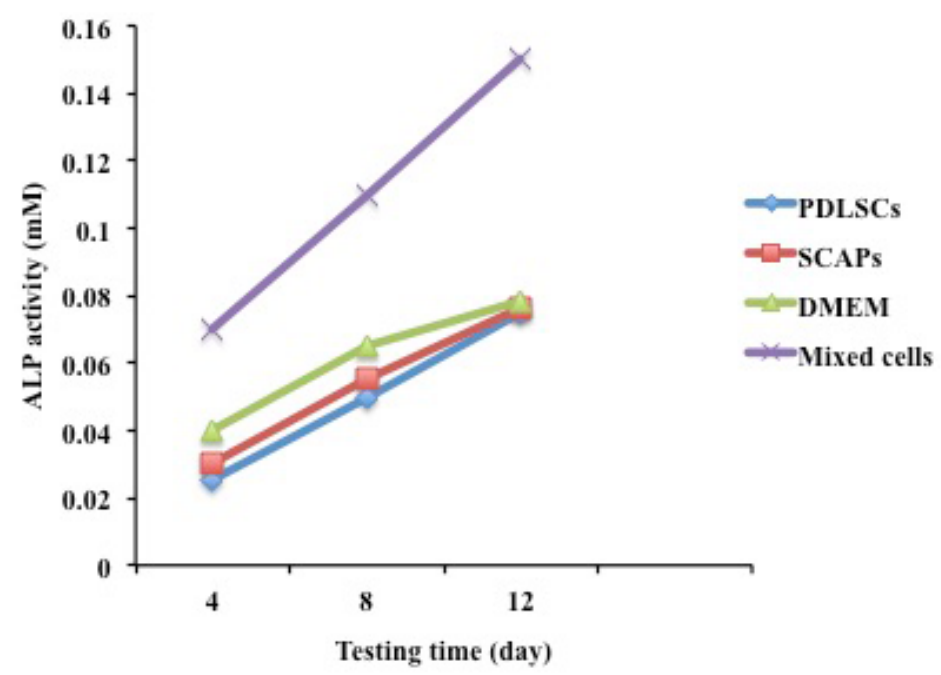

Figure 7. Alkaline phosphatase activities of the cell groups influenced by nicotinamide. DMEM, Dulbecco's modified Eagle's medium; PDLSCs, periodontal ligament stem cells; SCAPs, stem cells from apical papilla; ALP, alkaline phosphatase. 


\section{Differences in mineralization of cell groups treated with SIRT1 activator or inhibitor}

To evaluate the osteogenic differentiation of the three different cell groups (mixed cells, SCAPs, and PDLSCs) into a mineralized tissue, cells from each group were stained with Alizarin red solution following treatment with the SIRT1 activator or inhibitor. After 14 days, results showed a heavy calcium deposition in cells from the groups treated by SIRT1 activator, especially in the PDLSC group (Figure 8A), followed by the SCAP group (Figure 8B), and the mixed cell group (Figure $8 \mathrm{C}$ ). In comparison, a relatively smaller number of mineralized nodules developed in cells from the groups treated with SIRT1 inhibitor, as shown for the PDLSC (Figure 9A), SCAP (Figure 9B), and mixed cell groups (Figure 9C).
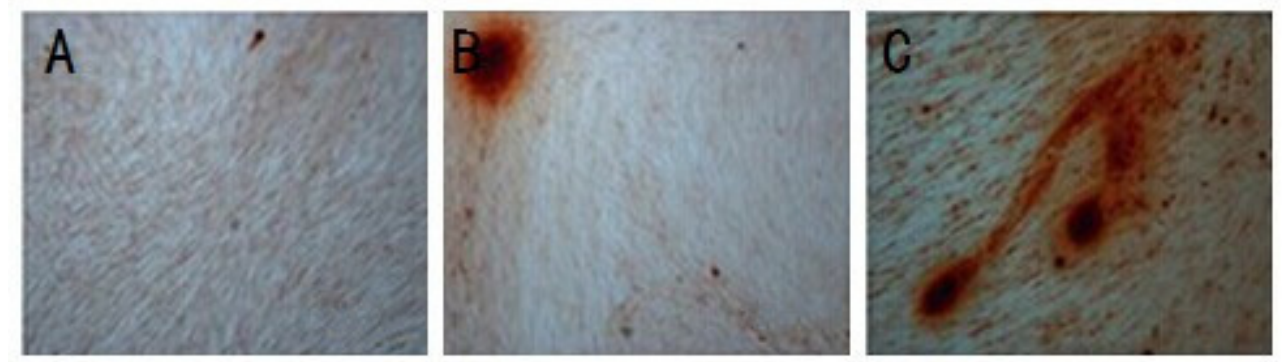

Figure 8. Mineralization potential with the SIRT1 activator as tested by Alizerin red solution staining. A. PDLSCs cultured in DMEM supplied with SIRT1 activator for 2 weeks showed few mineralized nodules. B. SCAPs cultured in DMEM supplied with SIRT1 activator for 2 weeks showed many mineralized nodules. C. PDLSCs co-cultured with SCAPs supplied with SIRT1 activator for 2 weeks showed an abundance of nodules. Scale bar, 100 um (A-C); DMEM, Dulbecco's modified Eagle's medium; PDLSCs, periodontal ligament stem cells; SCAPs, stem cells from apical papilla.
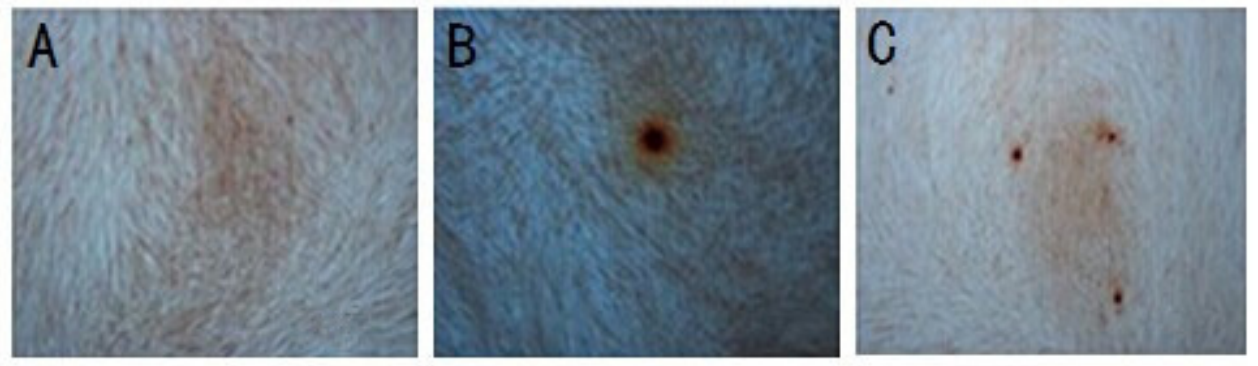

Figure 9. Mineralization potential with the SIRT1 inhibitor as tested by Alizerin red solution staining. A. PDLSCs cultured in DMEM supplied with SIRT1 inhibitor for 2 weeks showed few mineralized nodules. B. SCAPs cultured in DMEM supplied with SIRT1 inhibitor for 2 weeks showed decreased mineralized nodules. C. PDLSCs co-cultured with SCAPs supplied with SIRT1 inhibitor for 2 weeks showed decreased nodules. Scale bar, $100 \mu \mathrm{m}$ (A-C). DMEM, Dulbecco's modified Eagle's medium; PDLSCs, periodontal ligament stem cells; SCAPs, stem cells from apical papilla.

\section{qRT-PCR analysis of osteogenic differentiation markers}

On the 14th day of culture, markers of osteogenic differentiation were detected in the various cell groups by qRT-PCR, including ALP, osteopontin, osteocalcin, and bone sialoprotein. 
In the groups of cells with activated SIRT1, the markers of bone differentiation were significantly increased $(P<0.05$; Figure 10$)$, and in the groups having received treatment with the Sirtuin1 inhibitor, the expression levels of osteogenic differentiation markers were found to be significantly decreased $(P<0.05$; Figure 11).

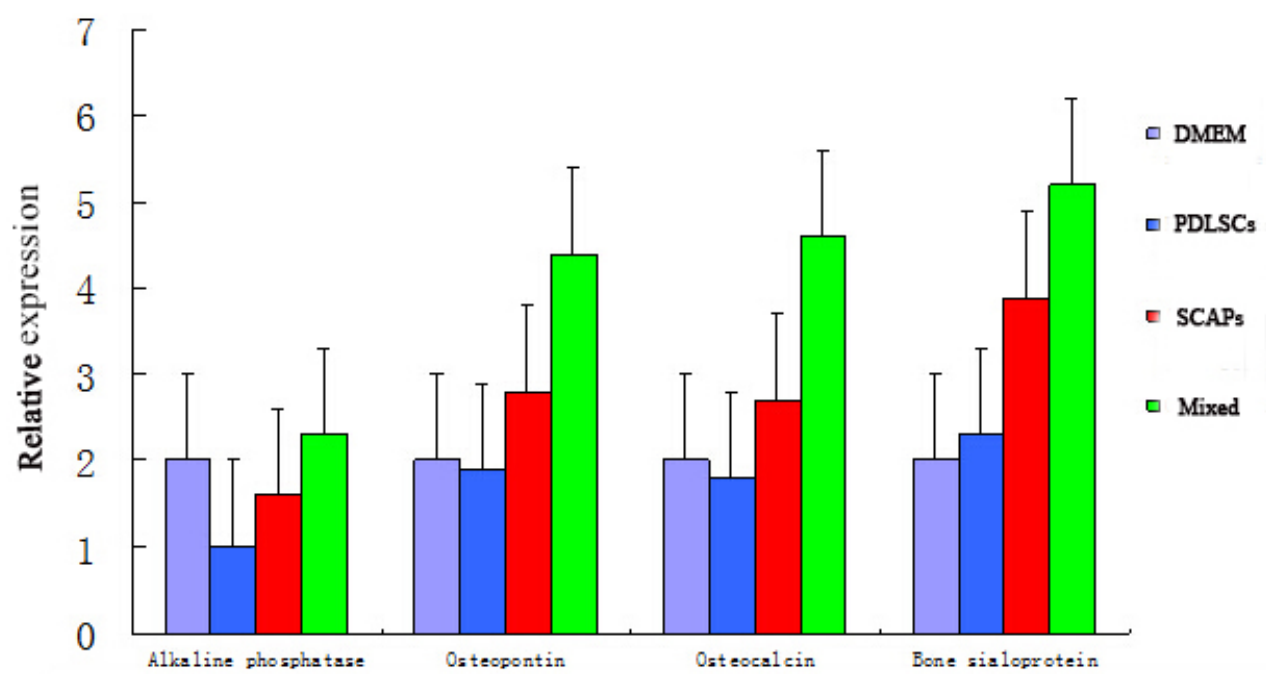

Figure 10. qRT-PCR results of osteogenic differentiation markers under the effect of the Sirtuin1 activator resveratrol. qRT-PCR, quantitative real-time PCR; DMEM, Dulbecco's modified Eagle's medium; PDLSCs, periodontal ligament stem cells; SCAPs, stem cells from apical papilla. Error bars mean \pm standard deviation. The internal reference represents $\beta$-actin. Samples were run in triplicate. Results were analyzed by SPSS 15.0. The expression level of osteogenic genes in the mixed group was higher than other groups; significant difference was shown $(P<0.05)$.

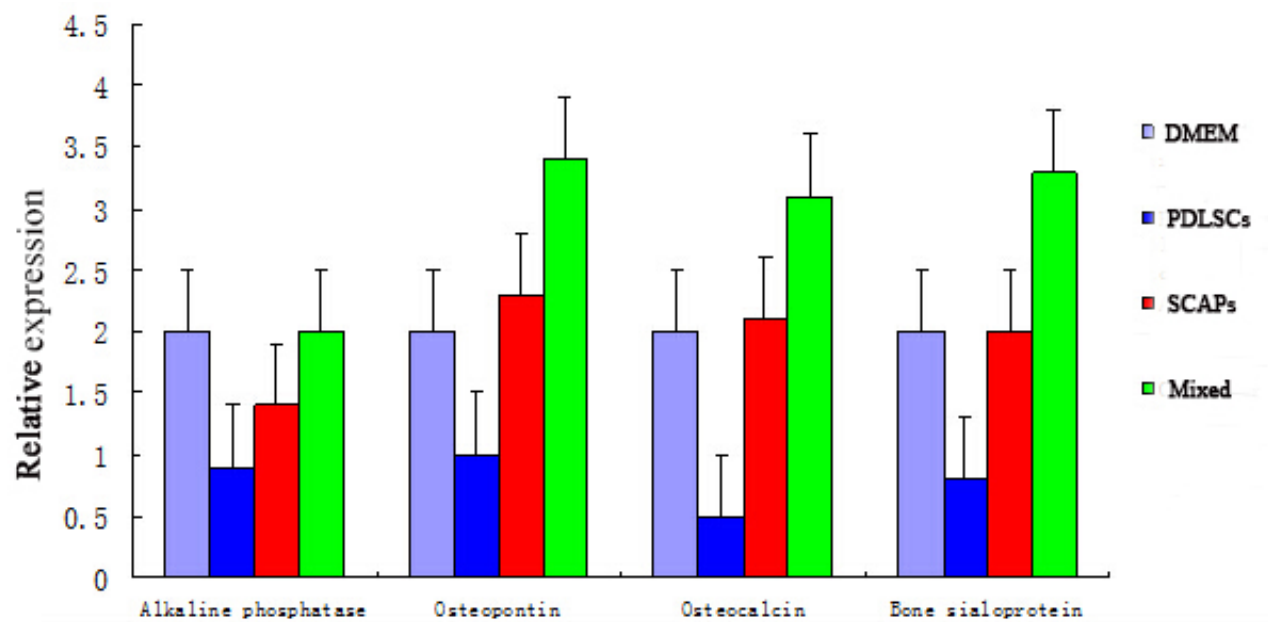

Figure 11. qRT-PCR results of osteogenic differentiation markers under the effect of Sirtuin1 inhibitor nicotinamide. qRT-PCR, quantitative real-time PCR; DMEM, Dulbecco's modified Eagle's medium; PDLSCs, periodontal ligament stem cells; SCAPs, stem cells from apical papilla. Error bars mean \pm standard deviation. The internal reference represents $\beta$-actin. Samples were run in triplicate. Results were analyzed by SPSS 15.0. The expression level of osteogenic genes in the mixed group was higher than other groups; significant difference was shown $(P<0.05)$. 


\section{DISCUSSION}

To our knowledge, periodontal ligament cells are principal functional cells, regenerating and reconstructing the periodontal tissues (Moshaverinia et al., 2014). The periodontal ligament is a source of pluri-potent cells and molecular factors that control cellular events in the periodontal tissues. Seo et al. in 2004 first discovered the stemness of periodontal ligament cells. PDLSCs have the ability of multi-directional differentiation. Osteogenic differentiation in particular has been verified in numerous reports (Houshmand et al., 2013; Wu et al., 2013). Recent studies have shown the property of heterogeneity in PDLSCs, which can enhance the formation of calcified nodules and the expression of markers of osteoblastic differentiation (Kim et al., 2012). In the developmental stage of the teeth, dental papilla originating from mesenchymal tissue has been proved to have the capacity of multiple differentiation, into both pulp and dentin (Bakopoulou et al., 2011). In 2006, Sonoyama et al. succeeded in isolating stem cells in the dental papilla from the undeveloped molar root apex. Anatomically, the dental papilla is located at the root tip close to the pulp. Biologically, there is a close relationship between apical papilla and the formation of the tooth root. SCAPs are considered to be potential candidates for bone/tooth tissue engineering because they are uncommitted and the multi-potent cells derived from them have the ability to become specialized mature cells (Huang et al., 2008). Many studies have demonstrated apexogenesis of immature teeth and continuous root development in teenagers suffering from endodontic diseases (Sonoyama et al., 2008).

Periodontal disease has been considered to be the leading cause responsible for human tooth loss clinically. Many research trials have been conducted in which reconstruction of the normal periodontal structure has been attempted, but these have been generally unsuccessful (Cochran et al., 2003; Miron et al., 2014). Periodontal regenerative measures, such as guided tissue regeneration, local application of enamel matrix proteins or other biological factors, and stem cell transplantation have, in contrast, been demonstrated to promote periodontal regeneration to a certain degree (Kwon et al., 2010; Akita et al., 2014). Although some progress has been made toward treating the effects of periodontal disease, these methods have various limitations. For example, guided tissue regeneration is difficult to operate and local application of enamel matrix proteins or other biological factors need some material to keep controlled release, but ideal support was not found yet (Floyd et al., 2014).

Osteoblastic differentiation and mineralization typically involve an initial period of cell proliferation and extracellular matrix biosynthesis, followed by cell differentiation. However, the mechanisms underlying this process are still unclear. Many signal pathways, biological factors, and small molecules have been investigated. In recent years, SIRT1 has been shown to be involved but has been rarely included in research involving stem cell differentiation (Pillarisetti, 2008). SIRT1 is an $\mathrm{NAD}^{+}$-dependent histone deacetylase, and a member of the Sirtuin family. Sirtuins are a family of proteins that are highly conserved from bacteria to complex eukaryotes, with a central region of approximately 250 amino acids (Picard et al., 2004). SIRT1 is found in mammalian cells, and is most homologous with the regulator of gene silencing, Sir2. Its physiological function is removal of an acetyl group to make the DNA chain more tightly condensed, leading to gene silencing. Several studies have demonstrated that SIRT1 regulates the cellular aging process by adjustment of the levels of p16, ERK, and p73 (Fulco et al., 2003; Haigis and Sinclair, 2010). SIRT1 also has been shown to be able to promote the formation of heterochromatin by regulation of histone methyltransferases. Resveratrol is considered to be an activator of SIRT1 and nicotinamide an inhibitor. Resveratrol and nicotinamide have been shown to promote or inhibit, respectively, the expression of markers of osteoblastic differentiation. The roles of resveratrol and nicotinamide in 
the differentiation of preadipocytes and neural stem cells have been preliminarily verified in several recent reports (Bai et al., 2008; Hisahara et al., 2008). Through such studies, SIRT1 has been demonstrated to be a potent regulator of cell differentiation although, as discussed above, the mechanisms underlying this regulation are still unclear. To date, little research has been performed to identify the roles of resveratrol and nicotinamide in the osteoblastic differentiation of dental stem cells.

In this study, our results firstly demonstrated that the two dental stem cell lines could each differentiate into osteoblasts that produced mineralized nodules and expressed early (ALP), intermediate (osteopontin), and late (osteocalcin) markers of differentiation. Furthermore, cooperative enhancement was found following co-culture of the two cell lines. The function of SIRT1 in the osteoblastic differentiation of PDLSCs and SCAPs was assessed and compared, and the key markers were tested and analyzed. Tests of the capacity of the cell lines for ALP activity demonstrated that the mixed cell lines showed the strongest ALP expression when treated with resveratrol, followed by the SCAP and then the PDLSC groups. In the groups treated with nicotinamide, the mixed group also showed the strongest relative expression of ALP activity, although at a decreased amount than that following resveratrol treatment; followed by the SCAP and PDLSC groups. With respect to mineralization, the PDLSCs co-cultured with SCAPs showed an abundance of nodules when treated with resveratrol as determined by Alizerin red staining, followed by the SCAP and then the PDLSC groups. Nodule areas were significantly higher in groups exposed to the SIRT1 activator than in those exposed to the inhibitor. In the groups treated with nicotinamide, most mineralization nodules appeared in the mixed group, followed by the SCAP and PDLSC groups, in descending order.

Our results also demonstrated that activation of SIRT1 by resveratrol stimulated osteoblastic differentiation in a cooperative enhancement manner, as assessed by the expression of ALP, osteopontin, osteocalcin, and bone sialoprotein mRNAs. Specifically, the capacity of osteoblastic differentiation increased from that of PDLSCs, to SCAPs, and through the mixed cell lines. Thus, the activation of SIRT1 was shown by two separate measures to promote the osteogenic process of dental stem cells, with the mixed cell lines having an additional, cooperative enhancement effect on osteogenesis, although the mechanism underlying this phenomenon remains unclear. In contrast, inhibition of SIRT1 by nicotinamide suppressed both mineralization and the expression of osteoblast markers. However, under this treatment condition the strongest relative expression of osteogenic markers was also observed in the mixed cell lines, despite the overall decreased expression levels, again highlighting the cooperative enhancement that occurred between the two cell lines. Overall, the results indicated that regardless of the stem cell group utilized, the activation of SIRT1 increased cell proliferation and enhanced the expression of osteoblastic differentiation markers. Similarly, inhibition of SIRT1 with nicotinamide generally suppressed cell proliferation and the expression of associated markers. SCAPs were shown to have a relatively stronger capacity for osteogenesis than PDLSCs, and the strongest capacity of osteogenesis appeared in the mixed cell group.

In summary, the results from this study demonstrated that SIRT1 is a potent regulator of cell proliferation and osteoblastic differentiation of human PDLSCs and SCAPs. Further research is still required, however, to elucidate the mechanisms underlying this differentiation process.

\section{ACKNOWLEDGMENTS}

Research supported by the Guang Dong Medical Scientific Research fund project (\#A2013294) and the fund of Ministry of Science and Technology in Guangzhou, Liwan District (\#20121214023). 


\section{REFERENCES}

Akita D, Morokuma M, Saito Y, Yamanaka K, et al. (2014). Periodontal tissue regeneration by transplantation of rat adiposederived stromal cells in combination with PLGA-based solid scaffolds. Biomed. Res. 35: 91-103. http://dx.doi.org/10.2220/ biomedres.35.91

Bäckesjö CM, Li Y, Lindgren U and Haldose'n LA (2007). Activation of Sirt1 decreases adipocyte formation during osteoblast differentiation of mesenchymal stem cells. J. Bone Miner. Res. 21: 993-1002.

Bai L, Pang WJ, Yang YJ and Yang GS (2008). Modulation of Sirt1 by resveratrol and nicotinamide alters proliferation and differentiation of pig preadipocytes. Mol. Cell Biochem. 307: 129-140. http://dx.doi.org/10.1007/s11010-007-9592-5

Bakopoulou A, Leyhausen G, Volk J, Tsiftsoglou A, et al. (2011).Comparative analysis of in vitro osteo/odontogenic differentiation potential of human dental pulp stem cells (DPSCs) and stem cells from the apical papilla (SCAP). Arch. Oral Biol. 56: 709-721. http://dx.doi.org/10.1016/j.archoralbio.2010.12.008

Beertsen W, McCulloch CA and Sodek J (1997). The periodontal ligament: a unique, multifunctional connective tissue. Periodontol. 2000 13: 20-40. http://dx.doi.org/10.1111/j.1600-0757.1997.tb00094.x

Blander G, Bhimavarapu A, Mammone T, Maes D, et al. (2009). SIRT1 promotes differentiation of normal human keratinocytes. J. Invest. Dermatol. 129: 41-49. http://dx.doi.org/10.1038/jid.2008.179

Cate AR (1996). The role of epithelium in the development, structure and function of the tissues of tooth support. Oral Dis. 2: 55-62. http://dx.doi.org/10.1111/j.1601-0825.1996.tb00204.x

Cochran DL, Jones A, Heijl L, Mellonig JT, et al. (2003). Periodontal regeneration with a combination of enamel matrix proteins and autogenous bone grafting. J. Periodontol. 74: 1269-1281. http://dx.doi.org/10.1902/jop.2003.74.9.1269

Floyd PD, Ide M and Palmer RM (2014). Clinical guide to periodontology: reconstructive periodontal treatment. Br. Dent. J. 216: 511-518. http://dx.doi.org/10.1038/sj.bdj.2014.354

Fulco M, Schiltz RL, lezzi S, King MT, et al. (2003). Sir2 regulates skeletal muscle differentiation as a potential sensor of the redox state. Mol. Cell 12: 51-62. http://dx.doi.org/10.1016/S1097-2765(03)00226-0

Haigis MC and Sinclair DA (2010). Mammalian sirtuins: biological insights and disease relevance. Annu. Rev. Pathol. 5: 253295. http://dx.doi.org/10.1146/annurev.pathol.4.110807.092250

Hayami T, Zhang Q, Kapila Y and Kapila S (2007). Dexamethasone's enhancement of osteoblastic markers in human periodontal ligament cells is associated with inhibition of collagenase expression. Bone 40: 93-104. http://dx.doi. org/10.1016/j.bone.2006.07.003

Hisahara S, Chiba S, Matsumoto H, Tanno M, et al. (2008). Histone deacetylase SIRT1 modulates neuronal differentiation by its nuclear translocation. Proc. Natl. Acad. Sci. U. S. A. 105: 15599-15604. http://dx.doi.org/10.1073/pnas.0800612105

Houshmand B, Behnia H, Khoshzaban A, Morad G, et al. (2013). Osteoblastic differentiation of human stem cells derived from bone marrow and periodontal ligament under the effect of enamel matrix derivative and transforming growth factor-beta. Int. J. Oral Maxillofac. Implants 28: e440-450. http://dx.doi.org/10.11607/jomi.te24

Huang GT, Sonoyama W, Liu Y, Liu H, et al. (2008). The hidden treasure in apical papilla: the potential role in pulp/dentin regeneration and bioroot engineering. J. Endod. 34: 645-651. http://dx.doi.org/10.1016/j.joen.2008.03.001

Kim SS, Kwon DW, Im I, Kim YD, et al. (2012). Differentiation and characteristics of undifferentiated mesenchymal stem cells originating from adult premolar periodontal ligaments. Korean J. Orthod. 42: 307-317. http://dx.doi.org/10.4041/ kjod.2012.42.6.307

Kwon DH, Bennett W, Herberg S, Bastone P, et al. (2010). Evaluation of an injectable rhGDF-5/PLGA construct for minimally invasive periodontal regenerative procedures: a histological study in the dog. J. Clin. Periodontol. 37: 390-397. http:// dx.doi.org/10.1111/j.1600-051X.2010.01546.x

Lee Y-M, Shin S-I, Shin K-S, Lee Y-R, et al. (2011). The role of sirtuin 1 in osteoblastic differentiation in human periodontal ligament cells. J. Periodontal Res. 46: 712-721. http://dx.doi.org/10.1111/j.1600-0765.2011.01394.x

Miron RJ, Wei L, Yang S, Caluseru OM, et al. (2014). Effect of an enamel matrix derivative on periodontal wound healing/ regeneration in an osteoporotic model. J. Periodontol. 85: 1603-1611. http://dx.doi.org/10.1902/jop.2014.130745

Moshaverinia A, Xu X, Chen C, Ansari S, et al. (2014). Application of stem cells derived from the periodontal ligament or gingival tissue sources for tendon tissue regeneration. Biomaterials 35: 2642-2650. http://dx.doi.org/10.1016/j. biomaterials.2013.12.053

Murakami S, Takayama S, Ikezawa K, Shimabukuro Y, et al. (1999). Regeneration of periodontal tissues by basic fibroblast growth factor. J. Periodontal Res. 34: 425-430. http://dx.doi.org/10.1111/j.1600-0765.1999.tb02277.x

Picard F, Kurtev M, Chung N, et al. (2004). Sirt1 promotes fat mobilization in white adipocytes by repressing PPAR-gamma. Nature 429: 771-776. http://dx.doi.org/10.1038/nature02583

Pillarisetti S (2008). A review of Sirt1 and Sirt1 modulators in cardiovascular and metabolic diseases. Recent Pat. Cardiovasc. Drug Discov. 3: 156-164. http://dx.doi.org/10.2174/157489008786263989 
Seo BM, Miura M, Gronthos S, Barthold PM, et al. (2004). Investigation of multipotent postnatal stem cells from human periodontal ligament. Lancet 364: 149-155. http://dx.doi.org/10.1016/S0140-6736(04)16627-0

Sonoyama W, Liu Y, Fang D, Yamaza T, et al. (2006). Mesenchymal stem cell-mediated functional tooth regeneration in swine. PLoS One 1: e79. http://dx.doi.org/10.1371/journal.pone.0000079

Sonoyama W, Seo BM, Yamaza T and Shi S (2007). Human Hertwig's epithelial root sheath cells play crucial roles in cementum formation. J. Dent. Res. 86: 594-599. http://dx.doi.org/10.1177/154405910708600703

Sonoyama W, Liu Y, Yamaza T, Tuan RS, et al. (2008). Characterization of the apical papilla and its residing stem cells from human immature permanent teeth: a pilot study. J. Endod. 34: 166-171. http://dx.doi.org/10.1016/j.joen.2007.11.021

Sumita Y, Honda MJ, Ohara T, Tsuchiya S, et al. (2006). Performance of collagen sponge as a 3-D scaffold for tooth-tissue engineering. Biomaterials 27: 3238-3248. http://dx.doi.org/10.1016/j.biomaterials.2006.01.055

Thesleff I and Mikkola M (2002). The role of growth factors in tooth development. Int. Rev. Cytol. 217: 93-135. http://dx.doi. org/10.1016/S0074-7696(02)17013-6

Wu Y, Yang Y, Yang P, Gu Y, et al. (2013). The osteogenic differentiation of PDLSCs is mediated through MEK/ERK and p38 MAPK signaling under hypoxia. Arch. Oral Biol. 58: 1357-1368. http://dx.doi.org/10.1016/j.archoralbio.2013.03.011

Yang ZH, Zhang XJ, Dang NN, et al. (2009). Apical tooth germ cell-conditioned medium enhances the differentiation of periodontal ligament stem cells into cementum/periodontal ligament-like tissues. J. Periodontal Res. 44: 199-210. http:// dx.doi.org/10.1111/j.1600-0765.2008.01106.x 